\title{
Conocimientos, actitudes y prácticas sobre sexualidad en estudiantes de secundaria del Colegio Adventista de Villa EI Salvador
}

Knowledge, attitudes and practices about sexuality in high school students of the Adventist School of Villa El Salvador

Ronald Alexis Nakayo Tiznado

ronaldnak@hotmail.com

Código ORCID: 0000-0003-4001-7344

Universidad Peruana Unión, Perú
Yvan Martín Balabarca Cárdenas

s.n.m.m@hotmail.com

Código ORCID: 0000-0002-2955-2816

Universidad Peruana Unión, Perú

Recibido: octubre 2020 / Arbitrado: noviembre 2020 / Publicado: enero 2021

RESUMEN

El objetivo del presente estudio fue determinar la relación de los conocimientos, actitudes y prácticas sobre la sexualidad de los estudiantes de secundaria del Colegio Adventista de Villa El Salvador, Lima, 2016. La metodología se fundamentó en el enfoque cuantitativo de tipo: descriptivo, exploratorio, correlacional, multivariado. Con un diseño: No experimental, Transversal. La población objeto de estudio fueron 380 estudiantes con una muestra No Aleatoria, No Probabilística formada por 195 y ampliada a 286 a criterio del investigador; adolescentes de los 5 grados de secundaria. Los resultados evidenciaron que aunque sólo el $40 \%$ de encuestados conoce muy poco a nada de la sexualidad juvenil motivo por el cual su actitud es de riesgosa a crítica aun los que refieren que mantienen una actitud sexual precavida también potencialmente están en riesgo todos estos aspectos lo corrobora la correlación. En conclusión no existe relación entre los conocimientos y las prácticas sexuales.

Palabras clave: Sexualidad; conducta sexual en riesgo, conocimiento sexual, práctica sexual, salud sexual, sexualidad saludable

The objective of this study was to determine the relationship of knowledge, attitudes and practices about sexuality of high school students of the Adventist School of Villa El Salvador, Lima, 2016. The methodology was based on the quantitative approach of type: descriptive, exploratory, correlational, multivariate. With a design: Non-experimental, Transversal. The study population was 380 students with a Non-Random, Non-Probabilistic sample consisting of 195 and expanded to 286 at the discretion of the researcher; teenagers in the 5th grade of secondary school. The results showed that although only $40 \%$ of respondents know very little about youth sexuality, which is why their attitude is from risky to critical, even those who report that they maintain a cautious sexual attitude are also potentially at risk all these aspects corroborate the correlation. In conclusion, there is no relationship between knowledge and

Keywords:

Sexuality; sexual behavior at risk; sexual knowledge; sexual practice, sexual health, healthy sexuality 


\section{INTRODUCCIÓN}

La organización mundial de la salud (OMS) denomina adolescentes al grupo de edad de 10 a 19, que atraviesan el periodo de cambio de la niñez a la edad adulta. (Guevara y Flores, 2004). Se valoriza como las etapas más cruciales de desarrollo, antes de la edad adulta, considerado como la etapa de transición más importantes del hombre, caracterizada por un ritmo acelerado de crecimiento y de cambios condicionada por diversos procesos biológicos iniciada en la pubertad, marcando el pasaje de la niñez a la adolescencia (OMS,2015).

Cortés, García, Monterrey, Fuentes y Pérez (2000) expresan que en la adolescencia temprana (10-15 años) aparecen los caracteres sexuales secundarios, hay fantasías sexuales, una gran despreocupación por los cambios puberales e incertidumbre acerca de su apariencia, es un período de turbulencia con gran ambivalencia afectiva. Asimismo, como complementa Monroe de Velazco (1990):

Que cada vez más existe un inicio precoz en las relaciones sexuales y su práctica sin protección, cambiar frecuente de parejas, uso compartido de agujas, consumo de alcohol además del uso de otras drogas que producen una inhibiciones sexuales y anular la capacidad juiciosa; todo estos aspectos hacen de este periodo una fase muy vulnerable a las infecciones de transmisión sexual (ITS), y dentro de ellas a la infección por VIH/SIDA. (p. 9)
En Latinoamérica, como refiere Ramos (2016),

... el tema del aborto en la región latinoamericana ha venido ocupando un lugar importante y creciente en el quehacer académico, el debate público y, en particular, en las agendas $y$ políticas públicas desde hace más de dos décadas". Resaltando la última publicación del Consorcio Latinoamericano contra el Aborto Inseguro, esta investigación presenta al aborto en América Latina y el Caribe. Donde indican que: "la tasa anual de aborto se mantuvo estable entre 2003 y 2008, permaneciendo en 31-32 abortos por 1,000 mujeres en edades de 15-19 años. Además de encontrarse en una controversia pública hoy existen numerosos casos de aborto en países de Latinoamérica y el Caribe. (p.1)

Es así como en estudios estadísticos de la Encuesta Demográfica de Salud Familiar ENDES (2014) mencionan que hay un vínculo muy estrecho entre el acceso a educación sexual en el momento indicado y la postergación del inicio sexual; un aspecto que se evidencia en las estadísticas de inicio sexual antes de los 15 años y que esta a su vez es 5 veces mayor en mujeres con menos de 7 años de escolaridad. Agrega, Rojas (2011) que "las Instituciones Educativas juegan un papel fundamental en la educación sexual de los adolescentes y que existen diversos factores que contribuyen a formar su propia cultura de su salud sexual" (p.3). 
El investigador antes mencionado explica que se ha observado en diferentes Centros de Salud y colegios de Lima que los adolescentes entre 14 a 17 años, ya usan algunas conceptualizaciones y mantienen actitudes que reflejan un manejo aceptables sobre temas de sexualidad y como cuidarse, sin embargo, aún se sigue observando casos de madres adolescentes, de abortos que muchas veces acaban en muertes siendo la mayor preocupación el VIH-SIDA.

Por su parte, Huapaya (2014) manifiesta que es una problemática juvenil que los adolescentes tengan conocimiento sobre sexualidad, sin embargo no practiquen una conducta sexual saludable, refiere el investigador que la actitud negativa y débil sobre el tema juega como motor principal a la hora de tomar decisiones de practicar una conducta riesgosa.

Frente a esta situación problemática nos planteamos la siguiente interrogante ¿Cuál es la relación de los conocimientos, actitudes y prácticas sobre sexualidad en estudiantes de secundaria del colegio adventista de Villa el Salvador, Lima, 2016?

Objetivo general: Determinar la relación de los conocimientos, actitudes y prácticas sobre sexualidad en estudiantes de secundaria del colegio adventista de Villa el Salvador, Lima, 2016.

Lo propuesto condujo a valorar como relevante dicha problemática, porque ofreció panorámicas argumentadas sobre la sexualidad en la comunidad educativa adolescente en Villa Salvador, los cuales permitieron llevar a cabo otros estudios más específicos en dicha población. En consecuencia, el aporte se focalizó en que los resultados ayudarán a plantear alternativas de mejoramientos a través de programas educativos sobre sexualidad desde el enfoque adventista $\mathrm{O}$ al contrario por medio de la UGEL aspecto que contribuirá al mejoramiento de la calidad del servicio estudiantil y el cuidado de la salud sexual, desarrollo, emprendimiento de los estudiantes.

En cuanto a la relevancia social, esta obedece a que permite intervenir, a través de acciones específicas, en los factores y conductas de riesgo de la sexualidad juvenil, así como contribuir al cuidado de la salud de la población, generando un perfil epidemiológico para futuras sociedades juveniles. En este sentido, el estudio también servirá a los padres, tutores y docentes, quienes conociendo los datos recabados tomen medidas preventivas en el cuidado de la salud sexual de sus hijos y alumnos.

Del mismo modo, los sectores de gobierno; sector educativo, salud y municipalidades podrán definir políticas públicas y normatividad preventiva sobre sexualidad que minimice el impacto de riesgo en la salud del adolescente en las comunidades educativas y población.

Con la finalidad de ahondar en el desarrollo exhaustivo de la problematización del objeto de estudio fue perentorio tomar en cuenta nociones y visiones de la temática que se presenta.

\section{El embarazo adolescente}

El embarazo en la adolescencia se ha considerado a lo largo de los siglos una problemática y preocupación a nivel mundial, pues a pesar de las múltiples acciones y estrategias, se advierte cada año 
un incremento de estos, lo que constituye un problema de salud que emerge en la época actual. El embarazo precoz y sus dos aristas o dilemas para la adolescente: me convierto en madre o aborto. Son las dos disyuntivas a las cuales se debe enfrentar el adolescente, siendo esto un desafío que se debe enfrentar en el terreno de la salud sexual y reproductiva, estableciéndose como reto en el terreno de la educación de la sexualidad para los profesionales encargados. Por otra parte, se recomienda la necesidad de conocer y evaluar con mayor objetividad la sexualidad de los chicos y chicas. Según las cifras establecidas, aproximadamente el $25 \%$ de la población mundial se ubica en las edades enmarcadas en la adolescencia (Quintero, Castillo y Romero, 2011).

Desde esta perspectiva preocupante es capital comprender las concepciones acerca de la interrupción del embarazo y sus implicaciones socioculturales.

\section{El aborto}

El aborto se define a partir del Diccionario la Lengua Española (2020) como la "Interrupción del embarazo por causas naturales o deliberadamente provocadas. Puede constituir eventualmente un delito" (s.n.). Otra idea es que: "El término aborto significa la interrupción del embarazo antes de que el feto sea viable. Lo más frecuente es que el aborto ocurra antes de la vigésima semana de gestación; si la interrupción tiene lugar entre el primero y tercer mes se habla de aborto precoz, denominándose aborto tardío entre el tercero y sexto mes de embarazo. Además, se añade que el aborto ha sido y es actualmente un método muy aplicado para regular la fecundidad, sobre todo en los países subdesarrollados. La OMS nos dice que casi la mitad de los abortos inducido que se realizan año tras año son inseguros. Estos se asocian con una considerable morbilidad materna, una de cada 5 mujeres que tuvieron un aborto inseguro, padecen una infección genital y alrededor del $13 \%$ de las muertes relacionadas con la gravidez se atribuyen a complicaciones provocadas por abortos inseguros (Velasco, 2010).

\section{Las relaciones sexuales a temprana edad}

Practicar las relaciones sexuales tiene muchos beneficios más que beneficios se vuelve una necesidad para muchas personas lo cual no está mal si se realiza de la manera adecuada, comenzando por la edad en la cual el cuerpo ya está preparado para este acto se dice que a los 13 años ya el cuerpo menstrua y está preparado para concebir pero se ha comprobado que una adolescente a esta edad podría provocarle desgarres y daños en su cuerpo por lo cual se llega a la conclusión que una edad adecuada seria a los 20 años ya que es menos propensa a tener daños tanto físicos como psicológicos, pero lo más recomendable según las escrituras bíblicas es que se practique durante el matrimonio lo cual es un consejo muy sabio y traería una mejor satisfacción (Fleiz et al, 1999).

Aunado a esta perspectiva, también forman parte de los basamentos del presente objeto de estudio las Teorías Cognitivas de la Sexualidad. El modelo cognitivo de Walen y Roth de 1987 (cp. Hyde, 1994) refiere que: 
nuestras percepciones y pensamientos determinan en gran medida nuestros sentimientos $y$ conductas. Así la forma como percibimos, rotulamos y evaluamos un evento sexual determina nuestras reacciones emocionales hacia tal evento y posiblemente también hacia otros aspectos relacionados. La importancia de esta conexión, entre aspectos cognitivos y emocionales, se puede apreciar claramente, entre otros temas, en los mecanismos psicológicos involucrados en algunos casos de disfunción sexual, y en las reacciones de las personas ante estímulos eróticos (p. 253)

Por otro lado, el modelo Secuencial de Byrne se desarrolló basándose sobre un paradigma clásico de estímulo- mediaciónrespuesta, el modelo secuencial de Byrne (cp. Becerra, 1985) quien describe que: "la conducta sexual se caracteriza mediante la identificación de las respuestas que se desean predecir, los estímulos externos que incrementan la probabilidad de aquellas respuestas, y los procesos internos que inician y median dichas respuestas" (s.n.) Por lo que este investigador focaliza una secuencia de tres fases: a) estimulación externa, b) procesos internos y c) conducta externa.

Asimismo también se conoce el modelo Cognitivo de Abramson, el cual entre diversos aspectos postula un esquema que se puede distinguir cuatro fases: a) factores de entrada; b) estructura cognitiva mediadora; c) claves o estímulos sexuales; y d) salida o expresión sexual (externa e interna). (Barra, 2002)

\section{MÉTODO}

Esta investigación fue de tipo correlacional, multivariado, el diseño no experimental de corte transversal. La población estuvo representada por 380 estudiantes de los 05 grados del nivel de secundaria de la Institución Educativa Adventista Villa Salvador. Para hallar la muestra se empleó el muestreo no aleatorio no probabilístico; para mayor representativa en esta población fue de 195; sin embargo el investigador amplio la muestra a 286 estudiantes.

La técnica empleada para recolectar datos fue una encuesta que evaluó conocimientos, actitudes y prácticas de la sexualidad en estudiantes. El instrumento fue diseñado por el investigador Mg. Angelo Huapaya en el 2014.

El instrumento se estructuró en dimensiones de conocimientos de 26 preguntas; actitudes 03 ítems (14 sub preguntas); y prácticas de 31 afirmaciones. Distribuidas de forma "salteada" con la finalidad de minimizar el sesgo de mentira y focalizar probables respuestas con índice de mentira. Para este último (detención de mentiras) se consideró la comparación de las respuestas de algunas preguntas focalizadas para el caso.

Para corroborar su validez en esta población que se caracteriza por su religiosidad se procedió a realizar la validez de constructo mediante la prueba KMO y Bartlett confirman que la validación de constructo es considerable. Donde la Medida Kaiser-Meyer-Olkin $=, 640$ lo que indica que la correspondencia de los ítems es considerable. $Y$ por otro lado el nivel de significancia de la Prueba de esfericidad de Bartlett $=, 000$. 
La confiabilidad original del año 2014 fue de: "alfa de Cronbach de 0,776", considerando algunas peculiaridades propias de la cultura adventista.

Luego se procedió a tomar una prueba piloto con 286 encuestados y se realizó un análisis estadístico del alfa de Cronbach que dio como resultado 0,844 lo que indica que este instrumento es confiable.

\section{RESULTADOS}

Los hallazgos del proceso investigativo se presentan a continuación en Tablas con su respectiva interpretación.

Tabla 1. Nivel de conocimientos sobre sexualidad de los estudiantes de la I.E.A. Villa Salvador que participaron del estudio de sexualidad 2016-II

\begin{tabular}{lcc}
\hline & Frecuencia & Porcentaje \\
\hline Conoce muy poco & 60 & 21,0 \\
Tiene un conocimiento regular & 49 & 17,1 \\
Tiene un conocimiento óptimo & 64 & 22,4 \\
Tiene excelente & 50 & 17,5 \\
No conoce nada sobre sexualidad & 63 & 22,0 \\
Total & 286 & 100,0 \\
\hline
\end{tabular}

En la Tabla 1 se observa que la mayoría de los estudiantes manifiestan tener un nivel de conocimiento óptimo sobre la sexualidad en $22.4 \%$. Mientras que la menor proporción tiene un conocimiento regular en $17.1 \%$. Y solo el $17.5 \%$ tienen un conocimiento excelente sobre sexualidad. Un $21 \%$ conoce muy poco y un $22 \%$ no conoce nada sobre sexualidad.

Tabla 2. Nivel de Prácticas sobre sexualidad de los estudiantes de la I.E.A. Villa Salvador que participaron del estudio de sexualidad 2016-II

\begin{tabular}{lcccc}
\hline & Frecuencia & Porcentaje & $\begin{array}{c}\text { Porcentaje } \\
\text { válido }\end{array}$ & $\begin{array}{c}\text { Porcentaje } \\
\text { acumulado }\end{array}$ \\
\hline Estado crítico & 63 & 22,0 & 22,0 & 22,0 \\
Práctica riesgosa & 72 & 25,2 & 25,2 & 47,2 \\
Práctica precavida & 44 & 15,4 & 15,4 & 62,6 \\
Práctica aceptable & 52 & 18,2 & 18,2 & 80,8 \\
Práctica saludable & 55 & 19,2 & 19,2 & 100,0 \\
Total & 286 & 100,0 & 100,0 & \\
\hline
\end{tabular}

En la Tabla 2 se observa que la mayoría de los estudiantes manifiestan una práctica riesgosa sobre la sexualidad en $25.2 \%$ y un $22 \%$ de forma crítica. Mientras que la menor proporción manifiestan una práctica precavida en $15.4 \%$. Y solo el $19.2 \%$ manifiesta una práctica saludable sobre sexualidad aunque $18,2 \%$ refiere que su prácticas son aceptables. 
Tabla 3. Nivel de Actitudes sobre sexualidad de los estudiantes de la I.E.A. Villa Salvador que participaron del estudio de sexualidad 2016-II

\begin{tabular}{lcccc}
\hline & Frecuencia & Porcentaje & $\begin{array}{c}\text { Porcentaje } \\
\text { válido }\end{array}$ & $\begin{array}{c}\text { Porcentaje } \\
\text { acumulado }\end{array}$ \\
\hline Actitud en estado crítico & 68 & 23,8 & 23,8 & 23,8 \\
Actitud riesgosa & 64 & 22,4 & 22,4 & 46,2 \\
Precavida & 48 & 16,8 & 16,8 & 62,9 \\
Actitud aceptable & 58 & 20,3 & 20,3 & 83,2 \\
Actitud saludable & 48 & 16,8 & 16,8 & 100,0 \\
Total & 286 & 100,0 & 100,0 & \\
\hline
\end{tabular}

En la Tabla 3 se observa que la mayoría de los estudiantes manifiestan una actitud en estado crítico y riesgoso sobre la sexualidad en $46.2 \%$. Mientras que la menor proporción manifiestan una actitud precavida $16.8 \%$ y saludable también $16.8 \%$. Y el $20.3 \%$ manifiesta una actitud aceptable sobre sexualidad.

Tabla 4. Determinar la relación de los conocimientos, actitudes y prácticas sobre la sexualidad de los estudiantes de la I.E.A. Villa Salvador que participaron del estudio de sexualidad 2016-II

\begin{tabular}{|c|c|c|c|c|c|}
\hline & & & Conocimiento & Actitud & Prácticas \\
\hline & & Coeficiente de correlación & 1 &,$- 240^{* *}$ & ,068 \\
\hline & Conocimiento & Sig. (bilateral) & &, 000 & ,255 \\
\hline & & $\mathrm{N}$ & 286 & 286 & 286 \\
\hline & & Coeficiente de correlación &,$- 240^{* *}$ & 1 &, $399^{* *}$ \\
\hline \multirow[t]{5}{*}{ Rho Spearman } & Actitud & Sig. (bilateral) &, 000 & &, 000 \\
\hline & & $\mathrm{N}$ & 286 & 286 & 286 \\
\hline & & Coeficiente de correlación & ,068 &, $399^{* *}$ & 1 \\
\hline & Prácticas & Sig. (bilateral) & ,255 &, 000 & \\
\hline & & $\mathrm{N}$ & 286 & 286 & 286 \\
\hline
\end{tabular}

*. La correlación es significativa al nivel 0,05 (bilateral).

**. La correlación es significativa al nivel 0,01 (bilateral).

En la Tabla 4 se observa que según el modelo estadístico Rho Spearman se reporta: en cuanto a la correlación de los conocimientos con las prácticas sexuales, el coeficiente $=.068$ un $p$ valor de $0.255>0,05$, indicando que la correlación es prácticamente nula y la correlación no es significativa. En cuanto a la correlación de los conocimientos con las actitudes sexuales, el coeficiente $=-.240$ un $p$ valor de $0.000<0,05$, indicando que la correlación de al menos en un $24 \%$ y significativa. En cuanto a la correlación de las actitudes con las prácticas sexuales, el coeficiente $=-.399$ un $p$ valor de $0.000<0,05$, indicando que la correlación es moderada de al menos en un $40 \%$ y significativa. 
Luego, habiendo evidencias suficientes para rechazar la hipótesis nula, se toma la decisión de aceptar la hipótesis de investigación en la cual se concluye que si existe relación directa y significativa entre el conocimiento y las actitudes así como también existe relación significativa entre las actitudes y las prácticas sexuales en los estudiantes. Sin embargo, no existe relación entre los conocimientos y las prácticas sexuales. Es decir que las prácticas saludables de sexualidad están relacionadas a la actitud positiva de los estudiantes más no con los conocimientos. $Y$ los conocimientos al menos están relacionados con la actitud de los estudiantes.

\section{CONCLUSIÓN}

El objetivo del presente estudio fue determinar la relación de los conocimientos, actitudes y prácticas sobre la sexualidad de los estudiantes de secundaria del colegio adventista de Villa el Salvador, Lima, 2016. Hallándose que existe correspondencia directa aunque y significativa entre el conocimiento y las actitudes así como también existe relación moderada y significativa entre las actitudes y las prácticas sexuales en los estudiantes.

Sin embargo, no existe relación entre los conocimientos y las prácticas sexuales.

Es decir que las prácticas saludables que estos adolescentes ejercen está implicada de forma moderada por la actitud que tengan sobre la sexualidad y en este caso se puede verificar a través de los resultados descriptivos el $62,6 \%$ mantienen prácticas de riesgo (considerando a precavida como riesgosa) en su comportamiento sexual tan similar que el $62,9 \%$ tiene una actitud riesgosa (considerando a precavida como riesgosa).
Un aspecto que explica que la actitud está implicando en que el adolescente tenga prácticas también riesgosas en su sexualidad.

Por otro lado, en cuanto a la relación indirecta baja y significativa entre conocimiento y actitud aunque sólo el $60 \%$ indique conocer de sexualidad de regular a más, mantienen una actitud muy riesgosa. Dicho de otro modo aunque sólo el $40 \%$ de encuestados conoce muy poco a nada de la sexualidad juvenil motivo por el cual su actitud es de riesgosa a crítica aun los que refieren que mantienen una actitud sexual precavida también potencialmente están en riesgo todos estos aspectos lo corrobora la correlación.

Finalmente, cabe resaltar que no existe correlación entre conocimientos y prácticas, esto demuestra que aunque los adolescentes manifiestan conocer de sexualidad de regular a un conocimiento óptimo sobre el tema sus prácticas de sexualidad se muestran de riesgosa a críticas en casi el 50\% (47,2\%) aunque si contabilizamos los que dicen tener una práctica precavida que también teóricamente están potencialmente en riesgo $(15,4 \%)$ este margen se elevaría a $62,6 \%$ un porcentaje que pone en esta crítico la población adolescente que estudia en dicho colegio.

Dicho comportamiento sexual es disonante con lo que expresa los adolescentes encuestados de su verdadera condición sexual; en la Tabla que describe la autovaloración de su salud sexual indicando que el $15,4 \%$ revela que su conducta sexual es crítica y un $29,4 \%$ dice ser precavido un aspecto que potencialmente también se tipifica como riesgo y un $22,7 \%$ no contestaron aspecto que indica que son más 
los adolescentes que están teniendo conducta promiscua.

Estos resultados focalizan la crisis en las actitudes de estos adolescentes en estudio, a la vez explican las altas tasas de embarazos adolescentes como bien lo enfatiza Restrepo, et al (2016) que el $84,4 \%$ de las adolescentes embarazadas tenían entre 16 y 19 años, y la edad mediana era de 17 años. El 61,2\% de ellas iniciaron su vida sexual a los 15 años. De la misma forma, Mamani Tagle y Silva Caballero (2012), quienes realizaron en Lima también su estudio sobre conductas sexuales de riesgo en estudiantes de secundaria, concluyeron lo siguiente: "Las conductas sexuales de riesgo se caracterizan por una alta prevalencia de estudiantes con relaciones coitales, inicio temprano de relaciones coitales, promiscuidad y ocasional o nula protección ante las Infecciones de transmisión sexual y embarazos no deseados" (p. 55).

\section{REFERENCIAS}

Barra, E. (2002). Psicología de la sexualidad. Concepción: Ed. Universidad de Concepción

Becerra, A. (1985). Psicología social y conducta sexual humana. En J.F. Morales, A. Blanco, C. Huici y J. Fernández, Psicología social aplicada. Bilbao: Desclée de Brouwer

Cortés, García, Monterrey, Fuentes y Pérez, (2000) Sida, adolescencia y riesgos. Rev. Cubana Med Gen Integr v.16 n.3 Ciudad de La Habana mayo-jun. 2000 pp. 1,17

ENDES Encuesta Demográfica de Salud Familiar (2014) [en línea] Recuperado de http://www.promsex.org/contents.php?i $\mathrm{d}=120$ p.15

Fleiz B. C, Villatoro V. J, Medina M. M., Alcántar M. E., Navarro G. C., Blanco J. J. (1999). Conducta sexual en estudiantes de la ciudad de México. Salud Mental 1999, 22. 14-19

Guevara Cachay, E., y Flores Aparco, D. C. (2004). Comportamiento sexual y uso de anticonceptivos modernos en adolescentes escolares con actividad coital. San Juan de Lurigancho

Huapaya F. G. (2014) "Conocimientos actitudes y practicas sobre sexualidad en una Universidad Privada de Lima, 2014". Tesis para optar el grado de maestría en Salud Pública, Universidad Peruana Unión, Perú

Hyde, J. (1994) Understanding human sexuality $\left(5^{\text {th }}\right.$ Ed.). New York: McGrawHill. p.253. Recuperado de http://dl.acm.org/citation.cfm?id=55423 4

Mamani T., Abel E. y Silva C., Jorge L. (2012). Consumo de alcohol y conductas sexuales de riesgo en estudiantes de secundaria de la Institución Educativa Nacional No72 San Martín de Porres UGEL N $N^{\circ} 03$

Monroe de Velazco, A. (1990). Expresión de la conducta sexual. Variantes sexuales. Salud sexualidad y adolescencia. Editorial PAX, México, DF, 185-90.

OMS. (2015) Desarrollo en la adolescencia. WHO [Internet]. 2015 [cited 2017 Apr 16]; Recuperado de http://www.who.int/maternal_child_adole scent/topics/

Quintero, P.P., Castillo R. N., Lazo C., Romero Y., et al (2011). Educación de la sexualidad en función de prevenir el embarazo en las adolescentes Rev Ciencias Médicas, 15(4):147-62

Ramos, S. (2016) Investigación sobre aborto en América Latina y el Caribe. Una agenda renovada para informar políticas públicas e incidencia (resumen ejecutivo) Estudios Demográficos y Urbanos, vol. 31, núm. 3, septiembre-diciembre, 2016, pp. 833- 860. El Colegio de México, A.C. Distrito Federal, México 
Real Academia Española. (Versión 23.4 en línea) Diccionario de la lengua española. Recuperado de https://dle.rae.es

Rojas L, R. (2011). Relación entre conocimientos sobre salud sexual y las conductas sexuales de riesgo en adolescentes de secundaria. I.E.M. Nuestra Señora de Montserrat Lima
2010. Tesis para optar el título profesional de licenciada en Enfermería. UNMSM, Lima, 2011. Recuperado de http://cybertesis.unmsm.edu.pe/bitstrea m/handle/20.500.12672/1302/rojas_Ir.pd $\mathrm{f}$ ? sequence $=1$ \&isAllowed $=\mathrm{y}$

Velasco, A., Cruz J. (2010). El aborto dentro del contexto social. 26(1):130-7 